\title{
TWO-NAIL FIXATION OF THE HIP
}

\author{
E. W. SOMERVille, Oxford, ENGLAND
}

From the Nuffield Orthopaedic Centre, Oxford

Osteoarthritis of the hip has a greatly varied incidence in different parts of the world. In Britain it has for many years presented one of the commonest and biggest problems in orthopaedic surgery - a problem which had shown little sign of solution until the introduction of intertrochanteric osteotomy by McMurray (1935). This operation entailed prolonged immobilisation, which excluded many elderly patients until the introduction of effective methods of internal fixation. More recently the total prosthesis, which may yet undergo many changes, has enabled us to deal remarkably effectively with the grossly damaged and distorted hip.

In the face of two operations of such obvious value as these it is easy to forget older operations of proved worth. Pseudarthrosis as described by Girdlestone (1945) and modified by Batchelor (1945) and Milch (1948) will always remain as a stand-by and last resort. Arthrodesis has become an uncommon operation confined almost entirely to young people.

Arthrodesis has been criticised on the grounds that it involves a prolonged period of immobilisation. It is difficult to obtain sound bony ankylosis, and if this is not achieved pain may persist and deformity develop. To reduce these difficulties Howard (1958) and Adams (1966) used the ischio-femoral arthrodesis by nail and graft, with or without a short hip spica, Charnley (1954) developed the central dislocation technique and Alvik (1950) used massive internal fixation. In spite of these modifications the operations have still been largely confined to younger patients.

Nevertheless, arthrodesis is the only procedure that provides a permanently painless and stable hip in unilateral osteoarthritis. If fusion could be obtained without external fixation and by some comparatively simple procedure, it might well remain an acceptable method of treatment.

With this in mind Watson-Jones (1938) and Burns (1939) advocated the use of a simple Smith-Petersen nail passed across the joint. This operation, initially successful, eventually failed because rotation was not adequately controlled and because it was often done on hips with good mobility. This paper is concerned with a modification of this technique in which two nails are used instead of one (Fig. 1).

\section{TECHNIQUE OF OPERATION}

The technique of operation is simple provided that the case has been selected with care. This procedure is not suitable for every hip. Hips with a good range of movement or with marked subluxation are generally unsuitable. Before operation is planned it should be demonstrated on the radiograph that two nails can be introduced at a suitable distance apart and can be sunk deeply into the pelvis across the joint. The presence of osteoarthritic cysts may be a hindrance or may make the operation impossible.

Positioning of the nails is of great importance and is the only part of the operation requiring skill. Good antero-posterior and lateral radiographs are essential. To get a good lateral view it is necessary to flex the other hip as shown in Figure 2. The object should be to sink both nails into strong bone in the pelvis, avoiding any cysts that may be present. Each nail should cross the joint by at least 2.5 centimetres to get a sound grip. The nails should be separated as far apart as possible where they cross the joint and should be slightly 
divergent. At first two standard Smith-Petersen nails were used but, after the development of a spontaneous fracture through the lower pin hole a pin and plate was substituted for the lower pin.

The method of alignment which has been found to be simple and satisfactory has been to introduce the first nail close to and parallel with the upper border of the neck of the femur. The introduction of the second nail is then easy, the alignment being taken from the first. The only estimation required is the length. If the pelvis is thin there seems to be no objection to this nail penetrating the inner cortex for a short distance.

The joint space may be widened during the introduction of the first nail if the pelvic bone is very hard. The gap must be closed before the second nail is inserted. Even if some gap remains,

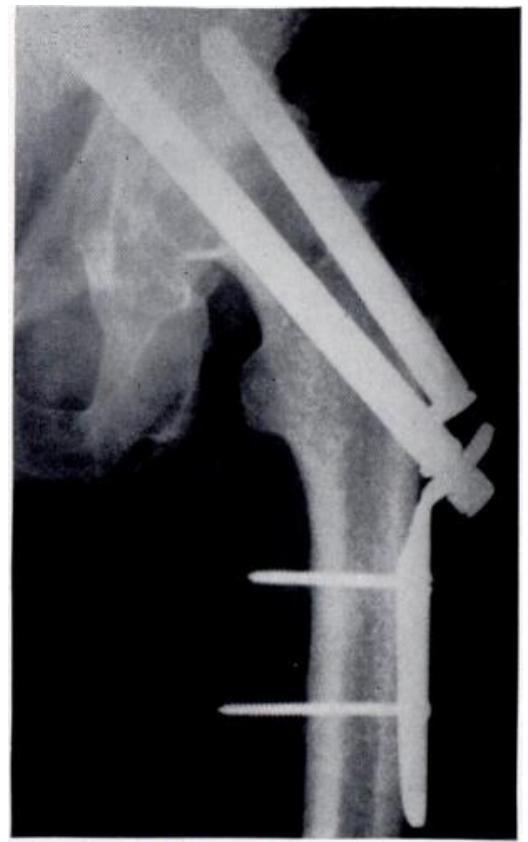

FIG. 1

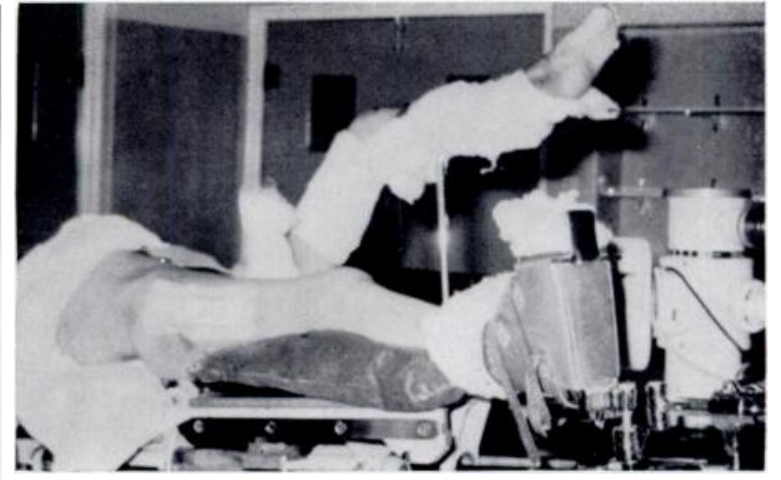

FIG. 2

Figs. 1 AND 2

Figure 1-Fixation of the hip with two triflanged nails. Figure 2-Position of the patient during the operation. The sound hip is flexed. The knee on the affected side is supported to avoid strain.

however, it will usually close with early weight-bearing. Certain precautions should be taken during the operation. It is always difficult to mobilise a knee when the hip is stiff, and it is important that no hyperextension strain shall fall on the knee during or after the operation; otherwise an effusion will develop and stiffness may persist and delay recovery.

The second precaution is to avoid widening the joint space and so producing instability. This may occur if a flexion contracture is forcibly corrected, when the femoral head may "hinge" in the acetabulum instead of rotating.

Most patients have been got up from bed and started walking with crutches on the second day after operation. It is common for the patients to walk with sticks by the end of the first week and they are encouraged to give up one or both sticks as soon as they want to.

\section{RESULTS}

The operation has been done twenty-seven times. Nineteen patients have been followed up for more than one and a half years, the longest follow-up being sixteen years and the shortest nine months. One patient died in diabetic coma within a month of operation and one could not be traced. 
The age at operation varied between nineteen and eighty-one. Nine patients were in their sixth decade, seven were in their seventh decade, three in their eighth, one patient was aged thirty-six and another forty-eight.

Of the nineteen patients followed up for more than eighteen months bony ankylosis as judged from trabeculation across the joint was considered to have been established in twelve. The absence of pain and the clinical absence of movement when under strain are not significant because these hips are rigidly fixed and painless from the time of the operation, and the absence of symptoms does not depend on bony ankylosis. In only one patient were the nails removed, confirming bony ankylosis.

In four patients there was some pain in the hip. In two it amounted to no more than an ache. In one patient the pain was described as severe: this patient was aged eighty-one

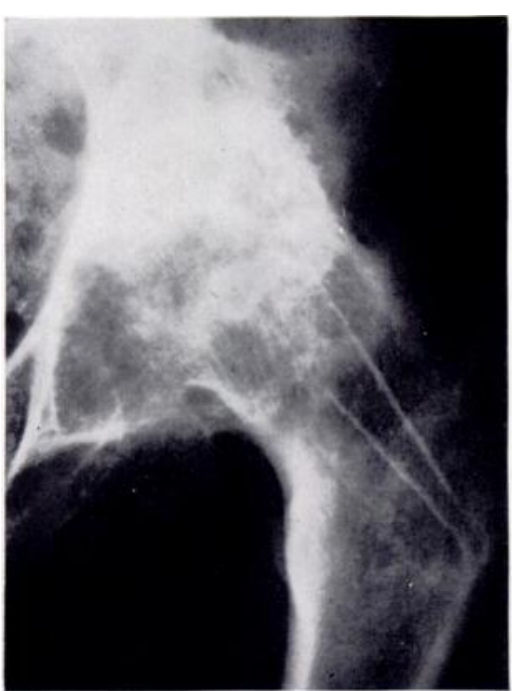

Fig. 3

Case 1 -Sound bony ankylosis five years after operation. at the time of operation. In one patient fracture occurred at the level of the lower nail ten days after operation, when the patient was leaving hospital. It was necessary to immobilise the hip in a spica for three months, after which union was sound and subsequently the hip fused.

In one patient aged nineteen, in whom the operation was performed for arthritis after traumatic dislocation of the hip with avascular changes, fusion did not occur and one nail broke. This man works in an engineering workshop and leads an active life, but complains of a slight ache.

None of the remaining fifteen patients made any complaint, whether or not the hip was fused. Twelve patients are doing hard work including housework, farm labouring and general labouring; one man is a steel erector.

Of the six patients who have been followed up for less than one and a half years four have returned to their previous occupation, though one has some discomfort in the knee which is rather stiff. In one patient return to work as a gardener was delayed because of a deep vein thrombosis.

Probably the best evaluation of the operation can be obtained from the case reports.

\section{ILLUSTRATIVE CASES}

Case 1-A man of sixty-one was seen in 1952 with severe pain in his left hip. At the age of twelve he had had a slipped upper femoral epiphysis. Treatment for that had been refused. He had been bedridden for many months. There was fixed flexion and adduction deformity of the hip of 20 degrees.

Two-pin arthrodesis of the hip was done in April 1952 and three days after operation the patient was allowed to stand. Ten days later he was walking well without pain and a week later he went home walking without help.

Five years later there was sound bony ankylosis and the pins were removed then because the ends were prominent and causing pain (Fig. 3). Sixteen years after operation he had no complaints regarding the left hip.

Case 2-A farmer aged fifty-six came with pain in the left hip that had been present for several years and was increasing. An operation for division of the psoas muscle was done in 1962 but produced only temporary improvement. In September 1964 there was severe pain in the hip and the patient could no longer manage his work. There was a fixed flexion contracture of 45 degrees and a fixed lateral rotation contracture of 20 degrees (Fig. 4).

Two-pin arthrodesis was done in September 1964; the patient walked two days after the operation and went home nine days later. In December 1964 he had no pain and was able to do his work. Four years after operation he had no complaints and was fully active (Fig. 5).

Case 3-A greenkeeper of fifty-six came with severe pain in the left hip that had been present for several years. He had had to give up work because of the restriction of activity. At the age of sixteen 
he had had a lesion of the hip that had been considered to be tuberculous, though proof was never obtained. The hip had a range of flexion of 90 degrees and of rotation of 25 degrees, but no abduction was permissible (Fig. 6).

Two-pin arthrodesis was done in October 1963; the patient walked two days after operation and went home four days later. He resumed work as a greenkeeper in January 1964 and by March that year could walk ten miles a day without help. Four years after the operation he had no symptoms from the hip (Fig. 7).

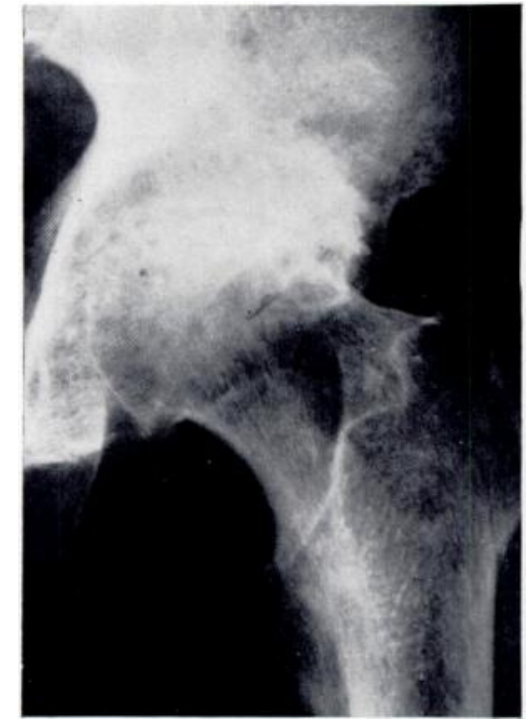

FiG. 4

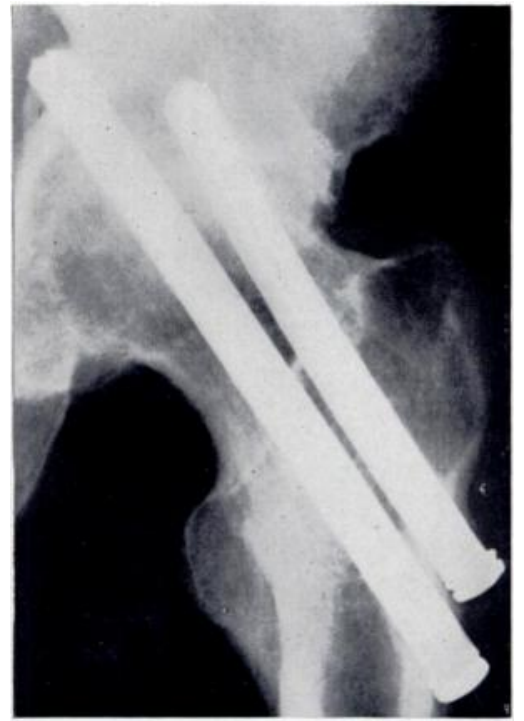

Fig. 5

Case 2. Figure 4-Radiograph of hip before operation. Figure 5-Two years after two-pin arthrodesis.

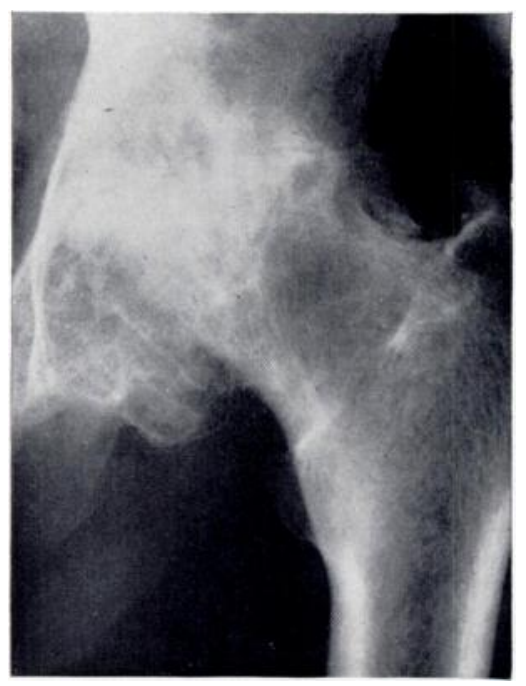

FIG. 6

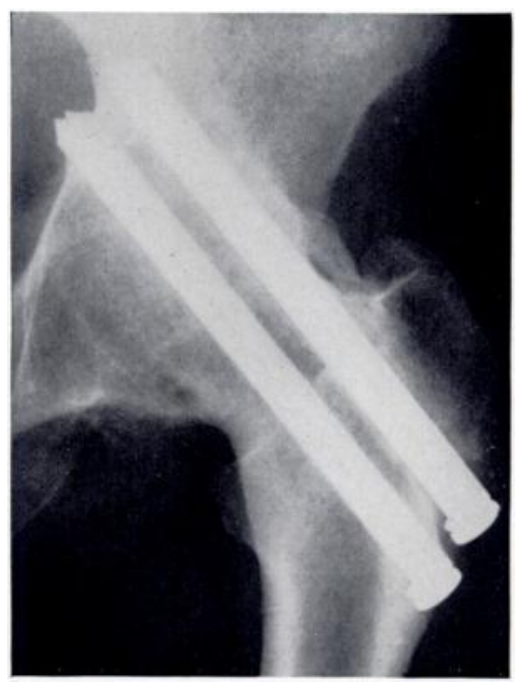

Fig. 7

Case 3. Figure 6-Before operation. Figure 7 -Two years after operation.

\section{DISCUSSION}

Obviously this method of arthrodesis has certain definite limitations, and cases should be carefully selected. The operation is performed for pain, and unless arthroplasty of the other

VOL. 51 B, NO. 4, NOVEMBER 1969 
hip is planned the condition must be unilateral. Ideally the less movement and the less deformity the better is the chance of success. Nevertheless, a certain amount of latitude is permissible. In one case the range of flexion was 90 degrees. In most the range was less than 45 degrees and in some it was a few degrees only.

The assessment of how much deformity is allowable is a matter of judgement and must depend on the activities of the patient. One patient had a fixed flexion deformity of 45 degrees. $\mathrm{He}$ was a farmer working single-handed and without this degree of flexion he would never have been able to milk his cows by hand. It presented no problem in relation to his other farming activities if he had a suitable raise on his boot.

In the case of a steel erector a fixed flexion deformity of 60 degrees was unacceptable: arthrodesis was combined with a posterior wedge osteotomy, so reducing the deformity to 30 degrees. The patient resumed his previous occupation without complaint. If arthrodesis

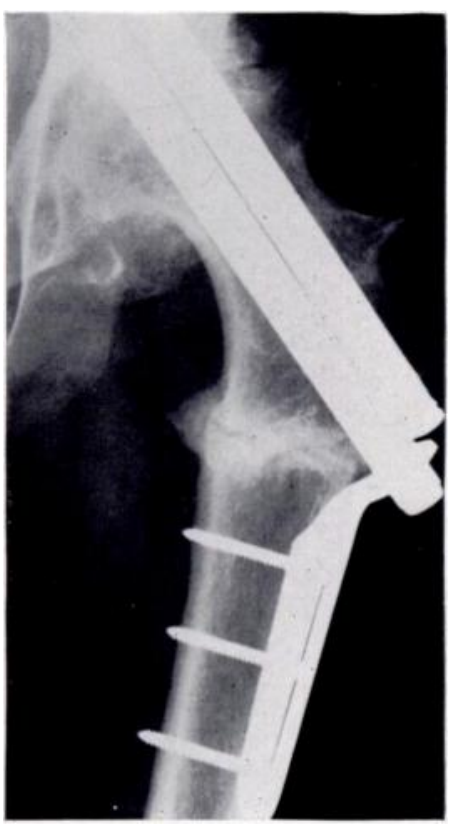

FIG. 8

Two-pin arthrodesis with corrective osteotomy. is combined with osteotomy the hip must be protected by a short spica until union is sound (Fig. 8).

Backache-The question arises of whether it is desirable to correct deformity when the hip of a middle-aged or elderly patient is fused for osteoarthritis. Osteoarthritis of the hip rarely develops without osteoarthritis developing in the spine. As the deformity of the hip slowly develops so the spine gradually adapts to compensate for it. If the hip deformity is corrected the adaptive changes in the spine must also be corrected: this leads to backache after arthrodesis. Backache may further be exacerbated by immobilisation of the spine for a long period. Correction of deformity should never be more than partial; fortunately it is usually possible to fix the hip in the position in which it lies. It is important not to allow correction to occur at the hip joint under the anaesthetic. Many of these hips are grossly deformed and forcible correction will result in separation of the joint surfaces; this decreases the stability which is so important in this operation.

All the patients in this series were questioned about backache and none would admit to any increase in backache after operation.

Bony ankylosis developed spontaneously in 63 per cent of the hips followed up for more than eighteen months after operation. This is not quite so good as in those series in which joint excision was combined with fixation, but the incidence in the whole series of only 10 per cent of patients having symptoms compares very favourably with the incidence in other series. The findings suggest that the introduction of two multiflange nails across the hip joint provides absolute immobilisation.

\section{SUMMARY}

1. A simple technique of arthrodesis of the hip is described.

2. Two triflanged nails are driven across the joint. The joint is not opened. No bone graft and no extensive fixation is used.

3. Symptoms were relieved in 90 per cent of patients and bony union was achieved in 63 per cent. Backache after operation was not a problem.

4. The period of hospitalisation was short and rapid return to heavy work was common. 


\section{REFERENCES}

Adams, J. C. (1966): Ischio-Femoral Arthrodesis. Edinburgh and London: E. \& S. Livingstone Ltd.

AlviK, I. (1950): Causes and Therapy of Arthrosis of Hip. Nordisk Medicin, 44, 1951.

BAtChelor, J. S. (1945): Excision of the Femoral Head and Neck in Cases of Ankylosis and Osteoarthritis of Hips. Proceedings of the Royal Society of Medicine, 38, 689.

BURNS, B. H. (1939): Fixation of the Osteo-arthritic Hip by Nailing. Lancet, i, 978.

Charnley, J. (1954): Stabilisation of the Hip by a Technique of Central Dislocation. Journal of Bone and Joint Surgery, 36-B, 692.

GiRDlestone, G. R. (1945): Discussion on Treatment of Unilateral Osteoarthritis of the Hip Joint. Proceedings of the Royal Society of Medicine, 38, 363.

Howard, R. C. (1958): "V" Arthrodesis of the Hip-joint. Proceedings of the Royal Society of Medicine, 51, 883.

MCMurRaY, T. P. (1935): Osteoarthritis of the Hip Joint. British Journal of Surgery, 22, 716.

МiLch, H. (1948): Resection-angulation Operation for Arthritis of the Hip. Bulletin of the Hospital for Joint Diseases, 9, 187.

Watson-Jones, R. (1938): Arthrodesis of the Osteo-arthritic Hip. Journal of the American Medical Association, $110,278$.

VOL. $51 \mathrm{C}$, NO. 4, NOVEMBER 1969 